PROCEEDINGS OF THE

AMERICAN MATHEMATICAL SOCIETY

Volume 125, Number 7, July 1997, Pages 2065-2072

S 0002-9939(97)03793-3

\title{
A CHANGE OF VARIABLES FORMULA FOR MAPPINGS IN BV
}

\author{
RUSTUM CHOKSI AND IRENE FONSECA
}

(Communicated by Jeffrey B. Rauch)

\begin{abstract}
A change of variables formula for mappings in $B V$ is obtained, where the usual jacobian is replaced by the determinant of the approximate differential.
\end{abstract}

\section{INTRODUCTION}

In this note, we use a result of Ambrosio [2] to extend to functions of bounded variation a well known change of variables formula for continuous mappings. In recent years, spaces of discontinuous mappings have proven to be useful in modeling deformations (displacements) of continua which exhibit defects and fracture (for example, see [4], [5], [8]). From the point of view of analysis, the natural space for the underlying deformation (or displacement) is that of functions of bounded variation, $B V$. Recently, De Giorgi and Ambrosio [6] proposed a smaller subclass for the study of some material instabilities, namely, the space of special functions of bounded variation, $S B V$.

Given $u \in B V\left(\Omega, \mathbb{R}^{N}\right)$, the functional gradient $\nabla u$ is the density of the absolutely continuous part (with respect to $N$-dimensional Lebesgue measure $\mathcal{L}^{N}$ ) of the distributional derivative $D u$. Whereas for a piecewise smooth mapping this functional gradient behaves, locally, like a true gradient, in general it may bear no structural resemblance to the classical curl free object. In fact, it has been shown that jump discontinuities may be dense, while $\nabla u$ may be any integrable tensor field (see [1]).

Suppose that $u \in B V\left(\Omega, \mathbb{R}^{N}\right)$ denotes the deformation of a body $\Omega$. What information about interpenetration of matter, i.e. the failure of a deformation to be one to one on sets of positive Lebesgue measure, can be inferred from either its full derivative or its functional derivative $\nabla u$ ? Suppose that we ignore the values of $u$ at the crack site (subset of $\Omega$ on which the mapping $u$ experiences jump discontinuities). Does the determinant of the functional derivative give any insight into this question? On the basis of the previous remarks, one is tempted to say no. However, it is well known that the functional derivative is an approximate differential almost everywhere. Using this fact, together with a Lipschitz approximation result due to Ambrosio [2], we prove that this information is still contained in the determinant

Received by the editors December 1, 1995 and, in revised form, January 30, 1996.

1991 Mathematics Subject Classification. Primary 26B10, 26B30, 49Q20.

Key words and phrases. Functions of bounded variation, maximal function, approximate differential. 
of the functional gradient, as long as one is willing to disregard the behavior of the mapping on a certain null set, which, in particular, encompasses the crack sites.

\section{Preliminaries}

Let $N$ be a positive integer, and let $\Omega$ be an open, bounded subset of $\mathbb{R}^{N}$. For $\mu$ a (vector-valued) Radon measure, we denote its total variation measure by $\|\mu\|$. $Q$ is the open unit cube $\left(-\frac{1}{2}, \frac{1}{2}\right)^{N}$, and $Q(a, r)$ is the open cube centered at $a$ with side length $r$, i.e., $Q(a, r)=a+r Q$. Also, $Q_{\nu}(a, r):=a+r Q_{\nu}$, where $Q_{\nu}$ is a unit cube centered at the origin with two of its faces normal to $\nu, \nu \in S^{N-1}:=$ $\left\{x \in \mathbb{R}^{N}:|x|=1\right\}$. As usual, $H^{N-1}$ denotes the $(N-1)$-dimensional Hausdorff measure.

We state some basic definitions and properties of smooth functions, of functions of bounded variation, $B V$, and of functions of special bounded variation, $S B V$. For more details, see Ambrosio [2], Evans and Gariepy [7], Fonseca and Gangbo [9], Morgan [11], and Ziemer [13].

Definition 2.1. Let $\phi: \Omega \rightarrow \mathbb{R}^{N}$ be a continuous function. For $x \in \Omega$ we set

$$
L_{\varepsilon}(y):=\frac{\phi(x+\varepsilon y)-\phi(x)}{\varepsilon},
$$

where $y \in B(0,1)$. Let $L: \mathbb{R}^{N} \rightarrow \mathbb{R}^{N}$ be a linear mapping.

(i) $L$ is is said to be the approximate differential of $\phi$ at $x$, and we write $L=$ (app) $d \phi_{x}$, if $L_{\varepsilon}$ converges to $L$ in measure on the ball $B(0,1)$, i.e.

$$
\lim _{\varepsilon \rightarrow 0} \mathcal{L}^{N}\left\{y \in B(0,1):\left|L_{\varepsilon}(y)-L(y)\right|>\delta\right\}=0
$$

for all $\delta>0$.

(ii) $L$ is called the weak differential of $\phi$ at $x$ if $L=(a p p) d \phi_{x}$ and if there exists a sequence $\left\{\varepsilon_{m}\right\}$ converging to 0 when $m$ tends to infinity such that $\left\{L_{\varepsilon_{m}}\right\}$ converges to $L$ uniformly on the sphere $S^{N-1}$.

(iii) If $\phi$ has a weak differential at $\mathcal{L}^{N}$ almost every point of $\Omega$, then we say that $\phi$ is weakly differentiable on $\Omega$.

Definition 2.2. A function $\phi: \Omega \rightarrow \mathbb{R}^{N}$ is said to satisfy the $N$-property if

$$
\mathcal{L}^{N}(\phi(E))=0
$$

for every $E \subset \Omega$ such that $\mathcal{L}^{N}(E)=0$.

Next, we introduce the space of functions of bounded variation.

Definition 2.3. A function $u \in L^{1}\left(\Omega ; \mathbb{R}^{N}\right)$ is said to be of bounded variation, $u \in B V\left(\Omega ; \mathbb{R}^{N}\right)$, if for all $i, j \in\{1, \ldots, N\}$ there exists a finite Radon measure $\mu_{i j}$ such that

$$
\int_{\Omega} u_{i}(x) \frac{\partial \varphi}{\partial x_{j}}(x) d x=-\int_{\Omega} \varphi(x) d \mu_{i j}
$$

for every $\varphi \in C_{0}^{1}(\Omega)$. The distributional derivative $D u$ is the matrix-valued measure with components $\mu_{i j}$. We denote by $\|D u\|$ the total variation of the gradient measure, i.e., $\|D u\|(\Omega):=\sum_{i=1}^{N}\left\|D u_{i}\right\|(\Omega)$, where

$$
\left\|D u_{i}\right\|(\Omega):=\sup _{\varphi}\left\{\int_{\Omega} u_{i} \operatorname{div} \varphi d x: \varphi \in C_{0}^{1}\left(\Omega, \mathbb{R}^{N}\right),|\varphi|_{\infty} \leq 1\right\} .
$$


We write

$$
D u=\nabla u \mathcal{L}^{N}+D_{s} u,
$$

where $\nabla u$ is the Radon-Nikodym derivative of $D u$ with respect to the $N$-dimensional Lebesgue measure $\mathcal{L}^{N}$, and $D_{s} u$ and $\mathcal{L}^{N}$ are mutually singular. Whenever there is no possibility of confusion, we abbreviate $\mathcal{L}^{N}(A)$ as $|A|$.

We denote by $S(u)$ the complement of the Lebesgue set of $u$; precisely, $x_{0} \notin S(u)$ if and only if there exists $\tilde{u}\left(x_{0}\right) \in \mathbb{R}^{N}$ such that

$$
\lim _{\varepsilon \rightarrow 0} \frac{1}{\left|B\left(x_{0}, \varepsilon\right)\right|} \int_{B\left(x_{0}, \varepsilon\right)}\left|u(y)-\tilde{u}\left(x_{0}\right)\right| d y=0 .
$$

Clearly $\tilde{u}$ is uniquely determined on $\Omega \backslash S(u)$.

Theorem 2.4. If $u \in B V\left(\Omega ; \mathbb{R}^{N}\right)$ then

(i) for $\mathcal{L}^{N}$ a.e. $x \in \Omega$

$$
\lim _{\varepsilon \rightarrow 0^{+}} \frac{1}{\varepsilon^{N}}\left\{\int_{Q(x, \varepsilon)}|u(y)-u(x)-\nabla u(x) \cdot(y-x)|^{\frac{N}{N-1}} d y\right\}^{\frac{N-1}{N}}=0 ;
$$

(ii) for $H^{N-1}$ a.e. $x \in S(u)$, there exists a unit vector $\nu(x) \in S^{N-1}$, normal to $S(u)$ at $x$, and there exist vectors $u_{-}(x), u_{+}(x) \in \mathbb{R}^{N}$, such that

$$
\begin{aligned}
& \lim _{\varepsilon \rightarrow 0^{+}} \frac{1}{\varepsilon^{N}} \int_{\left\{y \in Q_{\nu(x)}(x, \varepsilon):(y-x) \cdot \nu(x)>0\right\}}\left|u(y)-u_{+}(x)\right|^{\frac{N}{N-1}} d y=0, \\
& \lim _{\varepsilon \rightarrow 0^{+}} \frac{1}{\varepsilon^{N}} \int_{\left\{y \in Q_{\nu(x)}(x, \varepsilon):(y-x) \cdot \nu(x)<0\right\}}\left|u(y)-u_{-}(x)\right|^{\frac{N}{N-1}} d y=0 ;
\end{aligned}
$$

(iii) for $H^{N-1}$ a.e. $x_{0} \in S(u)$

$$
\begin{aligned}
\lim _{\varepsilon \rightarrow 0^{+}} \frac{1}{\varepsilon^{N-1}} \int_{Q_{\varepsilon} \cap S(u)}\left|u_{+}(x)-u_{-}(x)\right| d H^{N-1}(x) & =\left|u_{+}\left(x_{0}\right)-u_{-}\left(x_{0}\right)\right| \\
& >0, \\
\text { where } Q_{\varepsilon}:=Q_{\nu\left(x_{0}\right)}\left(x_{0}, \varepsilon\right) . &
\end{aligned}
$$

In view of the latter theorem, $S(u)$ is called the singular set, or jump set of $u$. It is well known that $S(u)$ is $N-1$ rectifiable (for example, see [7]), i.e.,

$$
S(u)=\bigcup_{n=1}^{\infty} K_{n} \cup \mathcal{N},
$$

where $K_{n}$ is a compact subset of a $C^{1}$ hypersurface for each $n$, and $H^{N-1}(\mathcal{N})=0$.

If $u \in B V\left(\Omega ; \mathbb{R}^{N}\right)$, then the measure $D u$ may be represented as

$$
D u=\nabla u \mathcal{L}^{N}+\left(u_{+}-u_{-}\right) \otimes \nu H^{N-1}\lfloor S(u)+C(u),
$$

where $C(u)$ is the so-called Cantor part. The three measures in (2.1) are mutually singular: if $H^{N-1}(B)<+\infty$ then $\|C(u)\|(B)=0$, and there exists a Borel set $E$ such that $\mathcal{L}^{N}(E)=0$ and $\|C(u)\|(X)=\|C(u)\|(X \cap E)$ for all Borel sets $X \subset \Omega$. A function $u \in B V\left(\Omega, \mathbb{R}^{N}\right)$ is said to be of special bounded variation if $C(u)=0$. We write $u \in S B V\left(\Omega ; \mathbb{R}^{N}\right)$. This subspace of $B V$ was introduced by De Giorgi and Ambrosio in [6]. 
Remark 2.5. In view of Definition 2.1 and Theorem 2.4 (i), if $u \in B V\left(\Omega, \mathbb{R}^{N}\right)$ then $\nabla u$ is an approximate gradient, and so (see [7], 6.13, Theorem 3) given $v \in$ $B V\left(\Omega, \mathbb{R}^{N}\right)$

$$
\nabla u=\nabla v \quad \mathcal{L}^{N} \text { a.e. } x \in\{u=v\} .
$$

\section{A CHANGE OF VARIABLES FORMULA AND APPLICATIONS}

Let $\mu$ be a finite, positive, scalar-valued Radon measure on $\Omega$. We define the maximal function of $\mu$ to be

$$
M(\mu)(x):=\sup \left\{\frac{\mu\left(B_{\rho}(x)\right)}{\left|B_{\rho}(x)\right|}: 0<\rho<\operatorname{dist}(x, \partial \Omega)\right\} .
$$

This notion was introduced explicitly by Ambrosio [2] for functions in $B V$ and is a generalization of the Hardy-Littlewood maximal function (see [12], and also [7] Section 6.62 for similar ideas). It can be shown that (see [2])

$$
|\{x \in \Omega: M(\mu)(x)>\lambda\}| \leq \frac{C(N) \mu(\Omega)}{\lambda},
$$

and so

$$
|\{x \in \Omega: M(\mu)(x)=+\infty\}|=0 .
$$

The proof of (3.1) is a simple consequence of the corresponding result obtained by Ambrosio ([2], Proposition 2.2) in the case where $\Omega$ is a ball, together with Besicovitch's Covering Theorem (see [2], Theorem 2.1).

Let $u \in B V\left(\Omega, \mathbb{R}^{N}\right)$ and let

$$
H(x):=M(\|D u\|)(x) .
$$

Define

$$
E_{\lambda}:=\{x \in \Omega: H(x)<\lambda\} \quad \text { and } \quad E:=\{x \in \Omega: H(x)<\infty\} .
$$

Note that $H$ is measurable and, by (3.2), $|\Omega \backslash E|=0$.

The following result was obtained by Ambrosio in [2] in the case where $\Omega$ is a ball, and the proof for an arbitrary open, bounded set $\Omega$ may be carried out exactly in the same way.

Theorem 3.1. Let $u \in B V\left(\Omega, \mathbb{R}^{N}\right) \cap L^{\infty}\left(\Omega, \mathbb{R}^{N}\right), \lambda>0$. Given $\rho>0$, define $\Omega_{\rho}:=\{x \in \Omega: \operatorname{dist}(x, \partial \Omega)>\rho\}$. Then there exists a Lipschitz function $u_{\lambda, \rho}:$ $\Omega_{\rho} \rightarrow \mathbb{R}^{N}$ such that

$$
\tilde{u}(x)=u_{\lambda, \rho}(x) \quad \text { for every } x \in\left(\Omega_{\rho} \cap E_{\lambda}\right) \backslash S(u) .
$$

The Lipschitz constant is bounded above by $C(N) \lambda+\frac{2|u|_{\infty}}{\rho}$.

We note that the maximal function $H$ is infinite at $H^{N-1}$ a.e. $x \in S(u)$. Hence in the latter theorem $\left(\Omega_{\rho} \cap E_{\lambda}\right) \backslash S(u)$ reduces to removing from $\Omega_{\rho} \cap E_{\lambda}$ a set of $H^{N-1}$ measure zero. See Remark 3.6 for more details.

Next we state a change of variables formula (see [9], Theorem 5.23) for a class of continuous functions, where, for a given set $A \subset \mathbb{R}^{N}, \sharp\{A\}$ stands for the number of elements of $A$ if $A$ is a finite set, and is $+\infty$ otherwise. 
Theorem 3.2. Let $\Omega \subset \mathbb{R}^{N}$ be an open, bounded set and assume that $\phi \in C\left(\Omega ; \mathbb{R}^{N}\right)$ has a weak differential almost everywhere, it has the $N$-property, and $\operatorname{det} \nabla \phi \in$ $L_{\text {loc }}^{1}(\Omega)$. If $v \in L^{\infty}\left(\mathbb{R}^{N}\right)$, then for every measurable set $A \subset \Omega$,

$$
\int_{A} v \circ \phi(x)|\operatorname{det} \nabla \phi(x)| d x=\int_{\mathbb{R}^{N}} v(y) N(\phi, A, y) d y,
$$

where $N(\phi, A, y)=\sharp\{x \in A: \phi(x)=y\}$.

We note that Lipschitz functions are weakly differentiable and satisfy the $\mathrm{N}$ property.

Finally, we state and prove a change of variables formula for $B V$. Roughly speaking, this is a change of variables formula for a mapping in $B V$ away from the crack site, in the unfractured zone.

Theorem 3.3. Let $u \in B V\left(\Omega, \mathbb{R}^{N}\right), v \in L^{\infty}\left(\mathbb{R}^{N}\right)$. Then for any measurable subset $A \subset \Omega$, the function $N(\tilde{u},(E \cap A) \backslash S(u), \cdot)$ is measurable in $\mathbb{R}^{N}$, and we have

$$
\int_{A} v \circ u(x)|\operatorname{det} \nabla u| d x=\int_{\mathbb{R}^{N}} v(y) N(\tilde{u},(E \cap A) \backslash S(u), y) d y,
$$

whenever one of the two integrals is meaningful.

Proof. It suffices to prove the result for $v \in L^{\infty}\left(\mathbb{R}^{N} ;[0,+\infty)\right)$. First assume that $u \in L^{\infty}$. Fix $\lambda>0$ and let $u_{\lambda}$ be the Lipschitz function given by Theorem 3.1 with $\rho=\frac{1}{\lambda}$. Let $A$ be a measurable subset of $\Omega$. By Theorem 3.2 and Remark 2.5,

$$
\begin{aligned}
\int_{\left(\Omega_{\rho} \cap E_{\lambda}\right) \cap A} v \circ u(x)|\operatorname{det} \nabla u| d x & =\int_{\left(\left(\Omega_{\rho} \cap E_{\lambda}\right) \cap A\right) \backslash S(u)} v \circ u_{\lambda}(x)\left|\operatorname{det} \nabla u_{\lambda}\right| d x \\
& =\int_{\mathbb{R}^{N}} v(y) N\left(u_{\lambda},\left(\left(\Omega_{\rho} \cap E_{\lambda}\right) \cap A\right) \backslash S(u), y\right) d y \\
& =\int_{\mathbb{R}^{N}} v(y) N\left(\tilde{u},\left(\left(\Omega_{\rho} \cap E_{\lambda}\right) \cap A\right) \backslash S(u), y\right) d y .
\end{aligned}
$$

By Theorem 5.5 in [9],

$$
N\left(u_{\lambda},\left(\left(\Omega_{\rho} \cap E_{\lambda}\right) \cap A\right) \backslash S(u), \cdot\right)=N\left(\tilde{u},\left(\left(\Omega_{\rho} \cap E_{\lambda}\right) \cap A\right) \backslash S(u), \cdot\right)
$$

is measurable. Thus, letting $\lambda \rightarrow \infty$ we conclude that $N(\tilde{u},(E \cap A) \backslash S(u), \cdot)$ is measurable, and by virtue of the Monotone Convergence Theorem we have

$$
\int_{A} v \circ u(x)|\operatorname{det} \nabla u| d x=\int_{\mathbb{R}^{N}} v(y) N(\tilde{u},(E \cap A) \backslash S(u), y) d y .
$$

It remains to remove the $L^{\infty}$ constraint on $u$. To this end let $u \in B V\left(\Omega, \mathbb{R}^{N}\right)$ be arbitrary and for each positive integer $n$ we define the Lipschitz function

$$
\phi_{n}(x):=\left\{\begin{array}{ccc}
x & \text { if } & |x| \leq n, \\
n \frac{x}{|x|} & \text { if } & |x|>n .
\end{array}\right.
$$

Let $A \subset B$ be measurable. For each $n$, we have

$$
\begin{aligned}
\int_{A} v\left(\phi_{n} \circ \tilde{u}(x)\right)\left|\operatorname{det} \nabla\left(\phi_{n} \circ u\right)\right| d x & =\int_{\mathbb{R}^{N}} v(y) N\left(\left(\phi_{n} \circ \tilde{u}\right),(E \cap A) \backslash S\left(\phi_{n} \circ u\right), y\right) d y \\
& =\int_{B(0, n)} v(y) N(\tilde{u},(E \cap A) \backslash S(u), y) d y,
\end{aligned}
$$


because $\phi_{n}\left(\mathbb{R}^{N}\right)=\bar{B}(0, n)$. By the chain rule formula, valid for the composition of Lipschitz functions with $B V$ functions (see [3]), and since $\operatorname{det} \nabla \phi_{n}(y)=0$ if $|y|>n$, we obtain

$$
\int_{A \cap\{|u(x)| \leq n\}} v(u(x))|\operatorname{det} \nabla u(x)| d x=\int_{B(0, n)} v(y) N(\tilde{u},(E \cap A) \backslash S(u), y) d y .
$$

Letting $n \rightarrow \infty$ on both sides of the above equation, we conclude (3.4) for arbitrary $u \in B V\left(\Omega, \mathbb{R}^{N}\right)$.

In the case where $v=1$, equation (3.4) is often referred to in the geometric measure theory literature as the Area Formula (see for example, see [7] or [11]). As an immediate consequence of (3.4), we have

Corollary 3.4. Let $u \in B V\left(\Omega, \mathbb{R}^{N}\right)$. Then $\operatorname{det} \nabla u \in L^{1}(\Omega)$ if and only if

$$
N(\tilde{u}, E \backslash S(u), y) \in L^{1}\left(\mathbb{R}^{N}\right) .
$$

In particular, if $\operatorname{det} \nabla u$ is not integrable then there is no representative of $u, \bar{u}$, for which $N(\bar{u}, \Omega, \cdot)$ is bounded and $\mathcal{L}^{N}(\bar{u}(\Omega))<+\infty$. It is well known that there are functions $u \in W^{1,1}\left(\Omega, \mathbb{R}^{N}\right) \subset B V\left(\Omega, \mathbb{R}^{N}\right)$ for which $\operatorname{det} \nabla u$ is not integrable. Corollary 3.4 characterizes this failure in terms of the integrability of the multiplicity function calculated on the "canonical" representative $\tilde{u}$, with domain set $E \backslash S(u)$.

Corollary 3.5. Let $u \in B V\left(\Omega, \mathbb{R}^{N}\right)$. Assume that $|\operatorname{det} \nabla u(x)|=1$ for a.e. $x \in \Omega$ and $|\tilde{u}(E \backslash S(u))|=|E \backslash S(u)|=|\Omega|$. Then for any measurable set $A \subset E \backslash S(u)$ we have

$$
|\tilde{u}(A)|=|A| .
$$

Proof. By Theorem 3.3, given a measurable set $A \subset E \backslash S(u)$, the function $N(\tilde{u}, A, y)$ is measurable, and so $\tilde{u}(A)=(N(\tilde{u}, A, \cdot))^{-1}(0,+\infty)$ is measurable. Using Theorem 3.3 with $v=1$ we have

$$
\begin{aligned}
|A| & =\int_{A}|\operatorname{det} \nabla u| d x \\
& =\int_{\mathbb{R}^{N}} N(\tilde{u}, A, y) d y \\
& \geq|\tilde{u}(A)| .
\end{aligned}
$$

Similarly,

$$
\left|A^{c} \cap(E \backslash S(u))\right| \geq\left|\tilde{u}\left(A^{c} \cap(E \backslash S(u))\right)\right| .
$$

If either (3.6) or (3.7) were strict inequalities then

$$
\begin{aligned}
|\Omega| & \geq|A|+\left|A^{c} \cap(E \backslash S(u))\right| \\
& >|\tilde{u}(A)|+\left|\tilde{u}\left(A^{c} \cap(E \backslash S(u))\right)\right| \\
& =|\tilde{u}(E \backslash S(u))|,
\end{aligned}
$$

contradicting the hypothesis.

Corollary 3.5 implies that the mapping $\tilde{u}$ cannot destroy volume but may create it, depending on whether the $N$-property holds on the complement of $E \backslash S(u)$. 
Remark 3.6. It follows from Theorem 2.4 that, setting

$$
\begin{aligned}
\mathcal{R}(u):=\left\{\begin{array}{l}
x \in S(u): u_{+}(x), u_{-}(x) \quad \text { exist }, \\
\end{array}\right. & \text { and } \left.\lim _{\varepsilon \rightarrow 0} \frac{\left(\left|u_{+}-u_{-}\right| H^{N-1}\lfloor S(u))\left(B\left(x_{0}, \varepsilon\right)\right)\right.}{\varepsilon^{N}}=+\infty\right\},
\end{aligned}
$$

then

$$
\mathcal{R}(u) \cap E=\emptyset, \quad \text { and } H^{N-1}(S(u) \backslash \mathcal{R}(u))=0 .
$$

Therefore, formula (3.4) may be reformulated as

$$
\int_{A} v \circ u(x)|\operatorname{det} \nabla u| d x=\int_{\mathbb{R}^{N}} v(y) N(\tilde{u},(E \cap A) \backslash(S(u) \backslash \mathcal{R}(u)), y) d y .
$$

Given that there is no natural way of extending $B V$ functions to sets of $H^{N-1}$ measure zero, we may rewrite this formula as

$$
\int_{A} v \circ u(x)|\operatorname{det} \nabla u| d x=\int_{\mathbb{R}^{N}} v(y) N\left(u^{*}, E \cap A, y\right) d y,
$$

where

$$
u^{*}(x):=\left\{\begin{array}{cl}
\tilde{u}(x) & \text { if } x \in \Omega \backslash(S(u) \backslash \mathcal{R}(u)), \\
0 & \text { if } x \in S(u) \backslash \mathcal{R}(u) .
\end{array}\right.
$$

In order to simplify even further the change of variables formula in such a way (3.4) may be written as (3.3), i.e. $E \cap A$ may be replaced by $A$, one has to redefine $u$ on the complement of the set $E$. Clearly, $\mathcal{L}^{N}\left(E^{c} \cup S(u)\right)=0$; setting $u$ to be 0 on $E^{c} \cup S(u)$, and denoting this representative of $u$ by $\hat{u}$, we would obtain

$$
\int_{A} v \circ \hat{u}|\operatorname{det} \nabla \hat{u}| d x=\int_{\mathbb{R}^{N}} v(y) N(\hat{u}, A, y) d y,
$$

for any $A \subset \Omega$ measurable, $v \in L^{\infty}\left(\mathbb{R}^{N}\right)$. However, there may be too much information, present in the original representation $\tilde{u}$, which is lost after performing this redefinition. For example, let $u$ be the Cantor-Vitali function. Here, $\nabla u=$ $0, S(u)=\emptyset$, and the range of $u$ is the entire unit interval. After redefinition, $N(\hat{u}, A, y)=0$ a.e. for every $A \subset[0,1]$ and $\mathcal{L}^{1}(\hat{u}([0,1]))=0$. This is an example where the $N$-property fails on the complement of $E$. Indeed, $E^{c}$ is the Cantor middle third set, and by (3.4) we have

$$
0=\int_{(0,1)} N(\tilde{u}, E, y) d y
$$

implying that $\mathcal{L}^{1}(\tilde{u}(E))=0$, and so $\mathcal{L}^{1}\left(\tilde{u}\left(E^{c}\right)\right)=1$.

Added comment. We are indebted to Luigi Ambrosio for valuable discussions on the subject matter, and for having referred us to the paper on "Area and Area Formula" by Giaquinta, Modica and Souček (see [10]) after this work was completed. In Theorem 1 of [10] an area formula is obtained for $L^{1}$ functions which are almost everywhere approximately differentiable, and hence, by Remark 2.5 , for $u \in B V\left(\Omega ; \mathbb{R}^{N}\right)$. In particular, it is proved that

$$
\int_{A} v(u(x))|\operatorname{det} \nabla u| d x=\int_{\mathbb{R}^{N}} v(y) N\left(\tilde{u},\left(\mathcal{D}_{\tilde{u}} \cap A\right) \backslash S(u), y\right) d y
$$


where $\mathcal{D}_{\tilde{u}}$ is the set of points of approximate differentiability of $\tilde{u}$, and whenever $v$ is the characteristic function of a measurable set. We remark that it follows from (3.4) and (3.8) that

$$
N\left(\tilde{u},\left(\mathcal{D}_{\tilde{u}} \cap A\right) \backslash S(u), y\right)=N(\tilde{u},(E \cap A) \backslash S(u), y)
$$

for $\mathcal{L}^{N}$ a.e. $y \in \mathbb{R}^{N}$; this identity does not seem to be easily deduced via a more direct approach.

\section{ACKNOWLEDGEMENTS}

The research of both authors was partially supported by the Army Research Office and the National Science Foundation, through the Center for Nonlinear Analysis (Carnegie Mellon University). The research of the first and second authors was partially supported by the National Science Foundation under Grant Nos. DMS9402763 and DMS-9500531 respectively.

\section{REFERENCES}

[1] Alberti, G. A Lusin type theorem for gradients. J. Funct. Anal. 100 (1991), 110-118. MR 92g:26018

[2] Ambrosio, L. On the lower semicontinuity of quasiconvex integrals in $S B V\left(\Omega, \mathbb{R}^{k}\right)$. Nonlinear Analysis Vol. 23, No. 3 (1994), 405-425. MR 95f:49011

[3] Ambrosio, L. and G. Dal Maso. A general chain rule for distributional derivatives. Proc. Amer. Math. Soc. 108 (1990), 691-702. MR 90j:26019

[4] Buttazzo, G. Energies on $B V$ and variational models in fracture mechanics. Preprint Dip. Mat. Univ. Pisa (1994).

[5] Choksi, R. and I. Fonseca. Bulk and Interfacial Energy Densities for Structured Deformations of Continua. To appear in Arch. Rat. Mech. Anal.

[6] De Giorgi, E and L. Ambrosio. Un nuovo tipo di funzionale del calculo delle vari azioni. Atti Accad. Naz. Lincei Rend. Cl. Sci. Fis. Mat. Natur. 82 (1988), 199-210. MR 92j:49043

[7] Evans, L. C. and R. F. Gariepy. Measure Theory and Fine Properties of Functions, CRC Press, 1992. MR 93f:28001

[8] Fonseca, I. and G. Francfort. Relaxation in $B V$ versus quasiconvexification in $W^{1, p}$; a model for the interaction between fracture and damage. Calc. Var. 4 (1995), 407-446. CMP 96:11

[9] Fonseca, I. and W. Gangbo. Degree Theory in Analysis and Applications. Oxford Univ. Press, 1995. MR 96k:47100

[10] Giaquinta, M., G. Modica and J. Souček. Area and the Area Formula. Rend. Sem. Mat. Fis. Milano LXII (1992), 53-87. MR 95g:49079

[11] Morgan, F. Geometric Measure Theory: A Beginners Guide. Academic Press, Boston, 1988. MR 89f: 49036

[12] Stein, E. Singular Integrals and Differentiability Properties of Functions. Princeton Univ. Press, Princeton, New Jersey, 1970.

[13] Ziemer, W. P. Weakly Differentiable Functions. Springer-Verlag, Berlin, 1989. MR 91e:46046

Courant Institute, New York University, New York, New York 10012

E-mail address: choksi@cims.nyu.edu

Department of Mathematics, Carnegie-Mellon University, Pittsburgh, Pennsylvania 15213

E-mail address: fonseca@andrew.cmu.edu 